\title{
Pattern statistics and Vandermonde matrices ${ }^{\star, \star \star}$
}

\author{
Massimiliano Goldwurm ${ }^{\mathrm{a}, *}$, Violetta Lonati ${ }^{\mathrm{a}}$ \\ ${ }^{a}$ Dipartimento di Scienze dell'Informazione - Università degli Studi di Milano, \\ Via Comelico 39, 20135 Milano, Italy.
}

\begin{abstract}
In this paper, we determine some limit distributions of pattern statistics in rational stochastic models. We present a general approach to analyze these statistics in rational models having an arbitrary number of strongly connected components. We explicitly establish the limit distributions in most significant cases; they are characterized by a family of unimodal density functions defined by means of confluent Vandermonde matrices.
\end{abstract}

Key words: Automata and formal languages, limit distributions, pattern statistics, rational formal series, Vandermonde matrices.

\section{Introduction}

This work presents some results on the limit distribution of pattern statistics. The major problem in this context is to estimate the frequency of pattern occurrences in a random text. This is a classical problem that has applications in several research areas of computer science and biology: for instance, it is considered in connection with the search of motifs in DNA sequences [17] while the earlier motivations are related to code synchronization $[10,11]$ and

\footnotetext{
ऋ Appeared in revised form in Theoretical Computer Science, vol. 356, 153-169, 2006. Preliminary version published in Proc. STACS 2005, 22nd Symposium on Theoretical Aspects of Computer Science, LNCS n. 3404, 680-692, Springer, 2005. ${ }^{\star \star}$ This research has been supported by Project MIUR PRIN 2005-2007 "Automata and Formal Languages: mathematical and applicative aspects".

* Corresponding author

Email addresses: goldwurm@dsi.unimi.it (Massimiliano Goldwurm), lonati@dsi.unimi.it (Violetta Lonati).
} 
approximated pattern-matching [13,22]. In a general probabilistic framework $[18,16,3]$, given one or more patterns, defined as strings over a finite alphabet $\Sigma$, and a probabilistic source $P$ generating words at random over $\Sigma$, one considers the number $X_{n}$ of occurrences of patterns in a word of length $n$ generated by $P^{(1)}$. Typical goals are the asymptotic evaluation of the moments of $X_{n}$, in particular its mean value and variance, its limit distribution, the local limit properties and the corresponding large deviations. The results depend in particular on the stochastic model $P$, which is usually assumed to be a Bernoulli model [11] or a Markovian model [18,16]. For instance, in [16] Gaussian limit distributions are obtained, for any regular set of patterns and any Markovian source $P$, under a primitivity hypothesis on the associated stochastic matrix.

In our paper, we assume the so-called rational stochastic model, introduced in [2], which includes the traditional Markovian model as a particular case. In our framework, the pattern is reduced to the single symbol $a$ while the text is a word of length $n$ over the alphabet $\{a, b\}$ generated at random according to a probability distribution defined by means of a rational formal series with nonnegative real coefficients and noncommutative variables $a, b$. Such a setting can simulate any Markovian source over an arbitrary finite alphabet $\Sigma$ for any regular set of patterns in $\Sigma^{*}[2]$.

Also in the rational stochastic models, Gaussian limit distributions are obtained under a primitive hypothesis, i.e. when the matrix associated with the rational formal series (counting the transitions between states) is primitive [2]. A complete study of the limit distributions is given in [5] in the bicomponent rational models, that is when the graph corresponding to the previous matrix consists of two strongly connected components.

Here, we present a general approach to the analysis of rational stochastic models with an arbitrary number of strongly connected components (called multicomponent models), explicitly establishing the limit distribution of the corresponding pattern statistics in most significant cases. The main result shows that such a limit distribution is related to the confluent Vandermonde matrices, a generalization of the classical Vandermonde matrices used in several research areas and in particular in Automatic Control Theory $[4,15]$.

The material we present is organized as follows. In Section 3 we recall the notion of confluent Vandermonde matrix and some of its properties; in particular, we show how this is related to the convolution of a finite set of sequences. In Section 4 we introduce a family of probability distributions defined by means of confluent Vandermonde matrices and establish their main properties. We call them Vandermonde distributions. In particular, we prove that their den-

$\overline{1 \text { Here, }}$ an occurrence is a position where a pattern ends in the text. 
sity functions are unimodal and we compute their characteristic functions. In Section 5 we start our analysis of pattern statistics and present the rational stochastic models, discussing the natural decomposition in strongly connected components; in particular, we introduce the notions of dominant component and main chain and show their role in the analysis of multicomponent models. In Section 6 we present our main result, which concerns the simple models (those with just one main chain which in addition only has primitive dominant components); in this case, assuming a mild variability condition on the dominant components, we determine the limit distribution of our pattern statistics showing that it is a Vandermonde distribution. Finally, in Section 7, we characterize the limit distributions for all simple models and provide a natural method to determine the limit distribution in the general case.

\section{Preliminary notions}

Generating functions represent the main tool we use in this study (see for instance [6] or [20, Chapter 3]). We recall that the (ordinary) generating function of a sequence $\left\{g_{n}\right\} \subseteq \mathbb{C}$ is the analytic function $g(w)$ that admits the Taylor expansion $g(w)=\sum_{0}^{+\infty} g_{n} w^{n}$ for every $w$ in an open neighbourhood of 0 . In our analysis we often have to evaluate the asymptotic growth of sequences having a rational generating function. To this end we make use of the following well-known properties that allow to extract informations on the growth of a sequence from the singularities of its generating function.

Let $g(w)$ be the generating function of a sequence $\left\{g_{n}\right\} \subseteq \mathbb{C}$; consider the radius of convergence $R$ of the power series $\sum_{0}^{+\infty} g_{n} w^{n}$ and assume $R$ is finite. We first observe that $g_{n}=\mathrm{O}\left(r^{-n}\right)$ for every real $r$ such that $0<r<R$. Moreover, let $\alpha_{1}, \alpha_{2} \ldots, \alpha_{j}$ be the singularities of $g(w)$ of modulus smaller than $T$, for some $T>R$. If all $\alpha_{i}$ 's are simple poles then $g_{n}=\sum_{i=1}^{j} c_{i} \alpha_{i}^{-n}+\mathrm{O}\left(\rho^{n}\right)$ for some $0<\rho<R^{-1}$ and some nonnull values $c_{i} \in \mathbb{C}, i=1, \ldots, j$. On the contrary, if each $\alpha_{i}$ is a pole of degree $k_{i}$, then $g_{n}=\sum_{i=1}^{j} c_{i} \alpha_{i}^{-n} n^{k_{i}-1}(1+$ $\mathrm{O}(1 / n))$ where $c_{i} \in \mathbb{C}$ is nonnull for every $i=1, \ldots, j$.

We finally recall that the product of two generating functions is the generating function of the convolution of the associated sequences. More generally, if $g^{(i)}(w)$ is the generating function of the sequence $\left\{g_{n}^{(i)}\right\}$ for each $i=1,2, \ldots, k$, then $f(w)=\prod_{i=1}^{k} g^{(i)}(w)$ is the generating function of the sequence $\left\{f_{n}\right\}$ such that, for every $n \in \mathbb{N}$,

$$
f_{n}=\sum_{n_{1}+\cdots+n_{k}=n} g_{n_{1}}^{(1)} g_{n_{2}}^{(2)} \cdots g_{n_{k}}^{(k)}
$$




\section{Confluent Vandermonde matrices}

Vandermonde matrices are defined by linear systems of equations whose solution yields the coefficients of polynomials of smallest degree with a given set of distinct roots [14]. When roots are associated with a given multiplicity an analogous system of equations can be defined that leads to a generalized version of Vandermonde matrix, called confluent Vandermonde matrix. That one plays a remarkable role in Automatic Control Theory [4]; in particular, its inverse is useful to compute the solutions of linear systems of differential equations [15]. In this section we recall the main properties of such matrices; our main goal is to present Proposition 3, which shows how the inverse of a confluent Vandermonde matrix can be used to compute the terms of the convolution of a family of sequences.

Given two integers $k, r$ such that $2 \leq r \leq k$, let $\left(v_{1}, v_{2}, \ldots, v_{r}\right)$ be a tuple of distinct complex numbers and let $\left(m_{1}, m_{2}, \ldots, m_{r}\right) \in \mathbb{N}^{r}$ be an associated tuple of multiplicities, such that $m_{1}+m_{2}+\cdots+m_{r}=k$ and $m_{i} \geq 1$ for each $i=1,2, \ldots, r$. Consider the monic polynomial

$$
D(x)=\prod_{\ell=1}^{r}\left(x-v_{\ell}\right)^{m_{\ell}}=x^{k}+a_{k-1} x^{k-1}+\cdots+a_{1} x+a_{0} .
$$

The confluent Vandermonde matrix associated with $D(x)$ is defined by $V=$ $\left[V_{1}\left|V_{2}\right| \cdots \mid V_{r}\right]$ where, for each $\ell=1,2, \ldots, r, V_{\ell}$ is the matrix of size $\left(k \times m_{\ell}\right)$ such that

$$
\left(V_{\ell}\right)_{h j}= \begin{cases}\left(\begin{array}{c}
h-1 \\
j-1
\end{array}\right) v_{\ell}^{h-j} & \text { if } j \leq h \\
0 & \text { otherwise }\end{cases}
$$

for every $h=1,2, \ldots, k$ and $j=1,2, \ldots, m_{\ell}$.

For instance if $r=2, m_{1}=3$ and $m_{2}=4$, then $V$ is given by

$$
V=\left(\begin{array}{ccc|cccc}
1 & 0 & 0 & 1 & 0 & 0 & 0 \\
v_{1} & 1 & 0 & v_{2} & 1 & 0 & 0 \\
v_{1}^{2} & 2 v_{1} & 1 & v_{2}^{2} & 2 v_{2} & 1 & 0 \\
v_{1}^{3} & 3 v_{1}^{2} & 3 v_{1} & v_{2}^{3} & 3 v_{2}^{2} & 3 v_{2} & 1 \\
v_{1}^{4} & 4 v_{1}^{3} & 6 v_{1}^{2} & v_{2}^{4} & 4 v_{2}^{3} & 6 v_{2}^{2} & 4 v_{2} \\
v_{1}^{5} & 5 v_{1}^{4} & 10 v_{1}^{3} & v_{2}^{5} & 5 v_{2}^{4} & 10 v_{2}^{3} & 10 v_{2}^{2} \\
v_{1}^{6} & 6 v_{1}^{5} & 15 v_{1}^{4} & v_{2}^{6} & 6 v_{2}^{5} & 15 v_{2}^{4} & 20 v_{2}^{3}
\end{array}\right) .
$$

In the special case when $m_{\ell}=1$ for every $\ell=1,2, \ldots r, V$ reduces to the 
standard Vandermonde matrix

$$
V=\left(\begin{array}{cccc}
1 & 1 & \ldots & 1 \\
v_{1} & v_{2} & \ldots & v_{k} \\
v_{1}^{2} & v_{2}^{2} & \ldots & v_{k}^{2} \\
\ldots & \ldots & \ldots & \ldots \\
v_{1}^{k-1} & v_{2}^{k-1} & \ldots & v_{k}^{k-1}
\end{array}\right) .
$$

It is well-known that $V$ always is nonsingular and that its determinant is $\prod_{1 \leq i<j \leq r}\left(v_{i}-v_{j}\right)^{m_{i} m_{j}}$.

\subsection{Inverse of a confluent Vandermonde matrix}

Some identities we use in subsequent sections concern the inverse of $V$ and especially the entries of its last column. An explicit expression for all entries of $V^{-1}$ is presented in [4, Eq. (9)]. Here, we recall that the last column of $V^{-1}$ is given by the vector

$$
\underline{w}=\left(w_{11}, w_{12}, \ldots w_{1 m_{1}}\left|w_{21}, w_{22}, \ldots w_{2 m_{2}}\right| \ldots \mid w_{r 1}, w_{r 2}, \ldots w_{r m_{r}}\right)^{T}
$$

where for every $\ell=1,2, \ldots r$ and $j=1, \ldots, m_{\ell}$, we have

$$
\frac{1}{D(x)}=\sum_{\ell=1}^{r} \sum_{j=1}^{m_{\ell}} \frac{w_{\ell j}}{\left(x-v_{\ell}\right)^{j}}
$$

and the following differential formula holds

$$
w_{\ell j}=\frac{1}{\left(m_{\ell}-j\right) !} \cdot \frac{d^{m_{\ell}-j}}{d x^{m_{\ell}-j}}\left[\frac{1}{\prod_{i \neq \ell}\left(x-v_{i}\right)^{m_{i}}}\right]_{\mid x=v_{\ell}} .
$$

Notice that for $x \neq v$ we have

$$
\frac{d^{n}}{d x^{n}}(x-v)^{-m}=(-1)^{n} n !\left(\begin{array}{c}
m+n-1 \\
m-1
\end{array}\right)(x-v)^{-m-n} .
$$

hence, by applying Leibniz differentiation rule

$$
\frac{d^{n}}{d x^{n}}\left(f_{1}(x) \cdot f_{2}(x) \cdots f_{r}(x)\right)=n ! \sum_{n_{1}+n_{2}+\cdots+n_{r}=n}\left(\prod_{i} \frac{1}{n_{i} !} \cdot \frac{d^{n_{i}}}{d x^{n_{i}}} f_{i}(x),\right)
$$

we get the following expression for every $w_{\ell j}$

$$
w_{\ell j}=(-1)^{m_{\ell}-j} \sum_{\sum_{i \neq \ell} n_{i}=m_{\ell}-j} \prod_{i \neq \ell}\left(\begin{array}{c}
n_{i}+m_{i}-1 \\
m_{i}-1
\end{array}\right)\left(v_{\ell}-v_{i}\right)^{-m_{i}-n_{i}} .
$$


Proposition 1 Let $D(x)$ be the polynomial defined by Equation (1) (with distinct $v_{\ell}$ 's). Consider the confluent Vandermonde matrix associated with $D(x)$ and let $\underline{w}$ be the vector defined in (3). Then, for every $s=1,2, \ldots k-1$ the following polynomial is identically null

$$
P_{s}(x)=\sum_{\ell=1}^{r} \sum_{j=1}^{\min \left(s, m_{\ell}\right)}\left(\begin{array}{l}
s-1 \\
j-1
\end{array}\right) w_{\ell j}\left(v_{\ell}-x\right)^{s-j} .
$$

Moreover, $P_{k}(0)=1$.

Proof. First notice that $P_{k}(0)=1$ and $P_{s}(0)=0$ for every $s=1,2, \ldots, k-1$. Indeed, such equalities can be written in matrix form as $V \cdot \underline{w}=(0, \ldots, 0,1)_{T}$, which holds true by definition of $V$ and $\underline{w}$. Now, fix an integer $1 \leq s \leq k-1$. Replacing $\left(v_{\ell}-x\right)^{s-j}=\sum_{h=j}^{s}\left(\begin{array}{c}s-j \\ h-j\end{array}\right) v_{\ell}^{h-j}(-x)^{s-h}$ in $P_{s}(x)$ we get

$$
P_{s}(x)=\sum_{\ell=1}^{r} \sum_{j=1}^{\min \left(s, m_{\ell}\right)} \sum_{h=j}^{s}\left(\begin{array}{l}
s-1 \\
h-1
\end{array}\right)\left(\begin{array}{c}
h-1 \\
j-1
\end{array}\right) w_{\ell j} v_{\ell}^{h-j}(-x)^{s-h} .
$$

Since the set $\left\{(h, j) \in \mathbb{N}^{2} \mid 1 \leq j \leq \min \left(s, m_{\ell}\right), j \leq h \leq s\right\}$ equals the set $\left\{(h, j) \in \mathbb{N}^{2} \quad 1 \leq h \leq s, 1 \leq j \leq \min \left(h, m_{\ell}\right)\right\}$, the previous expression can be written as

$$
P_{s}(x)=\sum_{h=1}^{s}\left(\begin{array}{l}
s-1 \\
h-1
\end{array}\right)(-x)^{s-h} \sum_{\ell=1}^{r} \sum_{j=1}^{\min \left(h, m_{\ell}\right)}\left(\begin{array}{c}
h-1 \\
j-1
\end{array}\right) w_{\ell j} v_{\ell}^{h-j}=\sum_{h=1}^{s}\left(\begin{array}{l}
s-1 \\
h-1
\end{array}\right)(-x)^{s-h} P_{h}(0)
$$

which is identically null by the previous reasoning.

Corollary 2 Let $V$ be the Vandermonde matrix defined in (2), where the $v_{\ell}$ 's are all distinct. Then, the entries of the last column of $V^{-1}$ are given by $c_{\ell}=\prod_{i \neq \ell}\left(v_{\ell}-v_{i}\right)^{-1}$ for $\ell=1,2, \ldots k$ and satisfy $\sum_{\ell} c_{\ell} v_{\ell}^{k-1}=1$, $\sum_{\ell} c_{\ell}\left(v_{\ell}-x\right)^{s-1}=0$ for every $s=1,2, \ldots k-1$.

\subsection{Multiple convolutions}

Confluent Vandermonde matrices are related to the properties of convolutions of families of sequences. More precisely, consider the rational function

$$
\frac{D(0)}{D(x)}=\prod_{\ell=1}^{r}\left(\frac{-v_{\ell}}{x-v_{\ell}}\right)^{m_{\ell}}=\prod_{\ell=1}^{r}\left(1-\frac{x}{v_{\ell}}\right)^{-m_{\ell}}
$$

and observe that each $\left(1-x / v_{\ell}\right)^{-m_{\ell}}$ is the generating function of $\left\{\left(\begin{array}{c}n+m_{\ell}-1 \\ m_{\ell}-1\end{array}\right) v_{\ell}^{-n}\right\}_{n}$. Therefore $D(0) / D(x)$ is the generating function of the sequence $\left\{g_{D}(n)\right\}_{n}$ defined by their convolution, i.e.,

$$
g_{D}(n)=\sum_{\sum_{\ell} n_{\ell}=n} \prod_{\ell=1}^{r}\left(\begin{array}{c}
n_{\ell}+m_{\ell}-1 \\
m_{\ell}-1
\end{array}\right) v_{\ell}^{-n_{\ell}}
$$


A key remark for the subsequent discussion is to notice that

$$
g_{D}(n)=\sum\left(v_{1}^{-n_{11}} \cdots v_{1}^{-n_{1 m_{1}}}\right) \cdot\left(v_{2}^{-n_{21}} \cdots v_{2}^{-n_{2 m_{2}}}\right) \cdots\left(v_{r}^{-n_{r 1}} \cdots v_{r}^{-n_{r m_{r}}}\right),
$$

where the sum is extended over all the $k$-tuples of nonnegative exponents $\left(n_{11}, n_{12}, \ldots, n_{r m_{r}}\right)$ whose sum equals $n$. In other words, $\left\{g_{D}(n)\right\}_{n}$ is the convolution of the sequences $\left\{v_{\ell}^{-n}\right\}_{n}$, each of them taken with multiplicity $m_{\ell}$.

Proposition 3 Let $V$ be the confluent Vandermonde matrix associated with the polynomial $D(x)$ defined by Equation (1) and assume that all roots $v_{\ell}$ 's are non-null. Also, let $g_{D}(n)$ be defined by Equation (7) for every $n \in \mathbb{N}$. Then,

$$
g_{D}(n)=D(0) \cdot \sum_{\ell=1}^{r} \sum_{j=1}^{m_{\ell}}\left(\begin{array}{c}
n+j-1 \\
j-1
\end{array}\right) \frac{w_{\ell j}}{\left(-v_{\ell}\right)^{j}}\left(v_{\ell}\right)^{-n}
$$

where the $w_{\ell j}$ 's are the entries of the last column of $V^{-1}$.

Proof. The generating function of the sequence $\left\{g_{D}(n)\right\}_{n}$ is given by $D(0) / D(x)$. Then, by equation (4) we have

$$
\sum_{n=0}^{\infty} g_{D}(n) x^{n}=\frac{D(0)}{D(x)}=D(0) \sum_{\ell=1}^{r} \sum_{j=1}^{m_{\ell}} \frac{w_{\ell j}}{\left(x-v_{\ell}\right)^{j}}
$$

and the result follows by applying

$$
\frac{1}{\left(x-v_{\ell}\right)^{j}}=\frac{1}{\left(-v_{\ell}\right)^{j}} \cdot \frac{1}{\left(1-x / v_{\ell}\right)^{j}}=\frac{1}{\left(-v_{\ell}\right)^{j}} \sum_{n=0}^{\infty}\left(\begin{array}{c}
n+j-1 \\
j-1
\end{array}\right)\left(v_{\ell}\right)^{-n} x^{n} .
$$

\section{Vandermonde distributions}

In this section we study the properties of a family of density functions naturally associated with confluent Vandermonde matrices. Let $r, k, v_{\ell}, m_{\ell}$ and $D(x)$ be defined as in Section 3. Consider the confluent Vandermonde matrix $V$ associated with $D(x)$ and the entries $w_{\ell j}$ 's of the last column of $V^{-1}$, for $\ell=1,2, \ldots, r$ and $j=1,2, \ldots m_{\ell}$. Now, assume that all $v_{\ell}$ 's are real and satisfy the relation $0 \leq v_{1}<v_{2}<\ldots<v_{r}$. Then, we define the real function

$$
f_{D}(x)= \begin{cases}0 & \text { if } x<v_{1} \\
(k-1) \sum_{\ell=h}^{r} \sum_{j=1}^{m_{\ell}}\left(\begin{array}{c}
k-2 \\
j-1
\end{array}\right) w_{\ell j}\left(v_{\ell}-x\right)^{k-j-1} & \text { if } v_{h-1} \leq x<v_{h} \\
0 & \text { for some } 1<h \leq r \\
& \text { if } x \geq v_{r}\end{cases}
$$


Its features mainly depend on the properties presented in Proposition 1. In particular notice that, for any $h \in\{2,3, \ldots r\}$, if $v_{h-1} \leq x<v_{h}$, then we obtain

$$
f_{D}(x)=-(k-1) \sum_{\ell=1}^{h-1} \sum_{j=1}^{m_{\ell}}\left(\begin{array}{c}
k-2 \\
j-1
\end{array}\right) w_{\ell j}\left(v_{\ell}-x\right)^{k-j-1} .
$$

Clearly, $f_{D}$ is continuously differentiable till the order $k-2$ in $\mathbb{R} \backslash\left\{v_{1}, \ldots, v_{r}\right\}$ and its $(k-2)$-th derivative is constant in each interval $\left(v_{\ell}, v_{\ell+1}\right), \ell=1, \cdots, k-$ 1. Moreover, using Proposition 1 , one can verify that, for any $\ell=1, \cdots, k-1$, the function $f_{D}$ is continuous at $v_{\ell}$ if and only if $m_{\ell} \leq k-2$ (note that this condition is true whenever $r \geq 3$ ). In general, $f_{D}$ is continuously differentiable at $v_{\ell}$ till the order $k-m_{\ell}-2$.

\subsection{Unimodal property}

Here, we prove that the function $f_{D}(x)$ defined above is nonnegative all over $\mathbb{R}$ and that, if $k \geq 3$, then $f_{D}$ is unimodal in $\left(v_{1}, v_{r}\right)$, that is there exists $t \in\left[v_{1}, v_{r}\right]$ such that $f_{D}(x)$ is strictly increasing in $\left[v_{1}, t\right]$ and strictly decreasing in $\left[t, v_{r}\right]$. Note that, if $f_{D}(x)$ is continuous (all over $\mathbb{R}$ ) then its unimodality in $\left(v_{1}, v_{r}\right)$ implies the existence of a unique (local) maximum in $\left(v_{1}, v_{r}\right)$.

To prove these properties, we consider two different cases: $k=r$ or $k>r$. If $k=r$, that is $m_{\ell}=1$ for every $\ell=1,2, \ldots, r$, then $f_{D}$ reduces to

$$
f_{D}(x)= \begin{cases}0 & \text { if } x<v_{1} \\ (k-1) \sum_{j=\ell}^{k} c_{j}\left(v_{j}-x\right)^{k-2} & \text { if } v_{\ell-1} \leq x<v_{\ell} \text { for some } 1<\ell \leq k \\ 0 & \text { if } x \geq v_{k}\end{cases}
$$

where $c_{\ell}=\prod_{i \neq \ell}\left(v_{\ell}-v_{i}\right)^{-1}$ for any $\ell=1,2, \ldots, r$. Note that, if $k=2$, then $f_{D}$ is the uniform density function over the interval $\left(v_{1}, v_{2}\right)$, while for $k=3$ we have the triangular distribution. The next proposition shows that, if $k=r \geq 3$, then $f_{D}$ is a unimodal function. The proof is based on Corollary 2 and makes use of the following lemma.

Lemma 4 Let $f: \mathbb{R} \rightarrow \mathbb{R}$ be a function admitting $j$-th derivative all over $\mathbb{R}$ for some $j \geq 1$. Also assume that, for some real values $a<b, f$ has $m$ zeros in the interval $(a, b)$ while $f(x)=0$ for each $x \leq a$ and each $x \geq b$. Then, for every $i=1, \ldots, j$, the $i$-th derivative of $f$ admits at least $m+i$ zeros in $(a, b)$.

Proof. We reason by induction on $i=1, \ldots, j$. If $i=1$, then consider the $m+1$ intervals determined by the zeros of $f$ in $[a, b]$. For each of them, say $\left(x_{1}, x_{2}\right)$, Rolle's Theorem guarantees that $f^{\prime}(x)=0$ for some $x \in\left(x_{1}, x_{2}\right)$. 
Now, assume $1<i<j$ and consider the $i$-th derivative of $f$, i.e. the function $g=f^{(i)}$. By the properties of $f$, we have $g(a)=g(b)=0$ and by the inductive hypotheses $g$ admits $m+i$ zeros in $(a, b)$. Therefore, by applying the previous argument to $g$, one proves that $g^{\prime}=f^{(i+1)}$ admits $m+i+1$ zeros in $(a, b)$.

Proposition 5 If $k=r \geq 3$, then $f_{D}$ is unimodal in $\left(v_{1}, v_{k}\right)$ and is nonnegative all over $\mathbb{R}$.

Proof. Using Corollary 2, one can prove that $f_{D}$ is strictly increasing in $\left(v_{1}, v_{2}\right)$ and strictly decreasing in $\left(v_{k-1}, v_{k}\right)$. In particular, this implies the property for $k=3$.

Now, let $k \geq 4$. Then, $f_{D}$ is continuously differentiable till the order $k-3$. Assume by contradiction that $f_{D}$ is not unimodal. Since the derivative $f_{D}^{\prime}$ is positive in $\left(v_{1}, v_{2}\right)$ and negative in $\left(v_{k}, v_{k-1}\right)$, this implies that $f_{D}^{\prime}$ necessarily vanishes in at least 3 points in the interval $\left[v_{2}, v_{k-1}\right]$. For $k=4$ this leads to a contradiction because $f_{D}^{\prime}$ is linear in $\left[v_{2}, v_{3}\right]$. For $k>4$, the function $f_{D}^{\prime}$ satisfies the hypotheses of Lemma 4 with $j=k-4, m=3, a=v_{1}, b=v_{r}$. As a consequence, the $(k-3)$-th derivative $f_{D}{ }^{(k-3)}$ of $f_{D}$ admits at least $k-1$ zeros in $\left(v_{1}, v_{k}\right)$, and this again leads to a contradiction. Indeed, $f_{D}{ }^{(k-3)}(x)$ is continuous all over $\mathbb{R}$, it is nonnull for $x \in\left(v_{1}, v_{2}\right) \cup\left(v_{k-1}, v_{k}\right)$, it is linear with respect to $x$ in each of the $k-3$ intervals $\left(v_{\ell}, v_{\ell+1}\right), \ell=2, \ldots k-2$, and hence it has at most $k-3$ many zeros in $\left(v_{1}, v_{k}\right)$.

Finally, since $f_{D}$ is positive in $\left(v_{1}, v_{2}\right) \cup\left(v_{k-1}, v_{k}\right)$ and admits a unique local maximum in $\left(v_{1}, v_{k}\right)$, then we can conclude that $f_{D}(x) \geq 0$ for every real $x$.

To prove that $f_{D}$ is unimodal also when $k>r$, we use the following lemma, which can be easily proved reasoning by contradiction [12].

Lemma 6 For every $n \in \mathbb{N}$, let $f_{n}: \mathbb{R} \rightarrow \mathbb{R}$ be a continuous function that admits a unique local maximum. If $\left\{f_{n}\right\}$ pointwise converges to a continuous function $f: \mathbb{R} \rightarrow \mathbb{R}$, then $f$ admits a unique local maximum, too.

We are now able to prove the complete property.

Proposition 7 If $k \geq 3$, then $f_{D}$ is nonnegative all over $\mathbb{R}$ and unimodal in $\left(v_{1}, v_{r}\right)$.

Proof. If $r=2<k$ then, by definition of $f_{D}$ and Eq. (6), one can show that

$$
f_{D}(x)=\frac{(k-1) !}{\left(m_{1}-1\right) !\left(m_{2}-1\right) !} \frac{\left(v_{2}-x\right)^{m_{1}-1}\left(x-v_{1}\right)^{m_{2}-1}}{\left(v_{2}-v_{1}\right)^{k-1}}
$$

for every $v_{1}<x<v_{2}$. It is easy to verify that $f_{D}$ is nonnegative and unimodal 
in $\left(v_{1}, v_{2}\right)$. Also note that $f_{D}$ is continuous unless $m_{1}=1$ or $m_{2}=1$ (and in these cases the only discontinuity point is $x=v_{2}$ or $x=v_{1}$, respectively).

If $r \geq 3$, then $f_{D}$ is continuous and we reason by induction on the integer $k-r$. If $k-r=0$, then the property is true by Proposition 5 . Thus, consider the case $k-r>0$. Then, there exists $\ell \in\{1,2, \ldots, r\}$ such that $m_{\ell}>1$. Recalling equation (8), we may assume $\ell=1$ without loss of generality. Given $0<\epsilon<v_{1}$, set $v_{0}=v_{1}-\epsilon$ and $m_{0}=1$ (if $v_{1}=0$, a similar discussion holds by setting $v_{0}=\epsilon$ for any $0<\epsilon<v_{2}$ ). Now, consider the polynomial

$$
D_{\epsilon}(x)=\frac{1}{x-v_{1}} \prod_{\ell=0}^{r}\left(x-v_{\ell}\right)^{m_{\ell}}
$$

and note that $D_{\epsilon}$ has $r+1$ distinct roots $v_{0}<v_{1}<\ldots<v_{r}$ with multiplicities such that $1+m_{1}-1+m_{2}+\ldots+m_{r}=k$. Thus, also $f_{D_{\epsilon}}$ is continuous and, by the inductive hypothesis, we know that $f_{D_{\epsilon}}$ is nonnegative in $\mathbb{R}$ and unimodal in $\left(v_{1}, v_{r}\right)$.

Let us study the pointwise convergence of $f_{D_{\epsilon}}(x)$ as $\epsilon$ goes to zero. If $x \geq v_{r}$, then $f_{D}(x)=f_{D_{\epsilon}}(x)=0$. If $x<v_{1}$ then, for $\epsilon$ small enough, $x<v_{0}$ and hence $f_{D_{\epsilon}}(x)=0=f_{D}(x)$. Finally, for any $h \geq 2$ and $v_{h-1} \leq x \leq v_{h}$ we have

$$
f_{D_{\epsilon}}(x)=\sum_{\ell=h}^{r} \sum_{j=1}^{m_{\ell}}\left(\begin{array}{l}
k-2 \\
j-1
\end{array}\right)\left(v_{\ell}-x\right)^{k-j-1} w_{\ell j}(\epsilon)
$$

where we use $w_{\ell, j}(\epsilon)$ to denote the entries of the last column of $V_{\epsilon}^{-1}, V_{\epsilon}$ being the confluent Vandermonde matrix associated with $D_{\epsilon}(x)$. Using equation (5), one can easily verify that $\lim _{\epsilon \rightarrow 0} w_{\ell j}(\epsilon)=w_{\ell j}$ for every $\ell \geq 2$. Thus, $f_{D_{\epsilon}}$ pointwise converges to $f_{D}$ all over $\mathbb{R}$, and the result follows by applying Lemma 6.

In Fig. 1 we show the plots of functions $f_{D}$ 's for three polynomials $D$, which present a rather regular behaviour. In these examples the number of distinct roots of $D$ (i.e. the value of $r$ ) is 2, 3 and 5 , respectively, and for each of them $f_{D}(x)$ is differentiable all over $\mathbb{R}$.
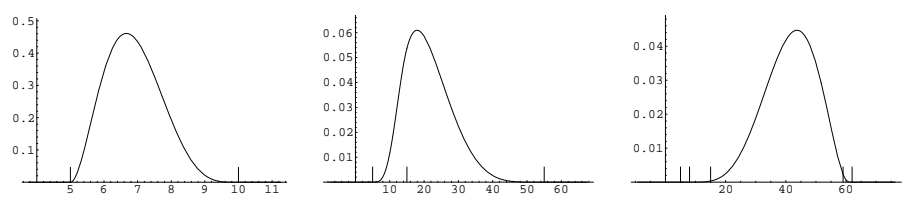

Fig. 1. Plots of function $f_{D}$ for $v=(5,10)$ and $m=(5,3), v=(5,15,55)$ and $m=(3,3,2), v=(5,8,15,59,62)$ and $m=(1,1,1,2,3)$, respectively. The vertical bars indicate the values of $v_{j}$ 's.

In Fig. 2 four special examples are illustrated which present irregular behaviours. In the first case there are two distinct roots of $D$ with multiplicity 4 and 1 , respectively, and the corresponding function $f_{D}$ is not continuous at the 
first root. In the other three examples $D$ has three distinct roots with different sets of multiplicities: in the case of simple roots $f_{D}$ is a triangular density function (see the second picture); if the array of multiplicities is $m=(1,4,1)$, then $f_{D}$ is continuous but not differentiable at the second root, while the same behaviour occurs at the first root if $m=(4,1,1)$.
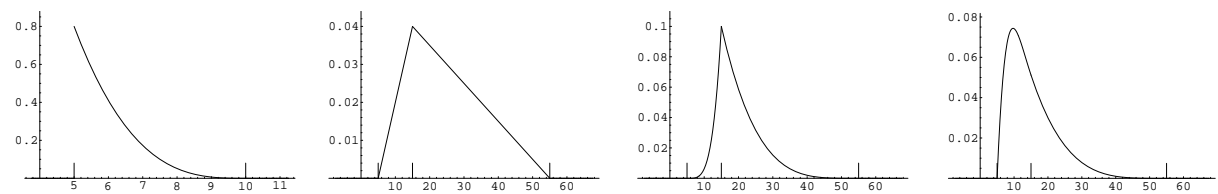

Fig. 2. Plots of function $f_{D}$ for $v=(5,10)$ and $m=(4,1), v=(5,15,55)$ and $m=(1,1,1), v=(5,15,55)$ and $m=(1,4,1), v=(5,15,55)$ and $m=(4,1,1)$, respectively. The vertical bars indicate the values of $v_{j}$ 's.

\subsection{Characteristic function}

Here, we prove that $f_{D}$ is a density function. Since it is nonnegative all over $\mathbb{R}$, it is sufficient to prove that $\int_{-\infty}^{+\infty} f_{D}(x) d x=1$. Further, we show that the characteristic function of $f_{D}$ is given by

$$
\Phi_{D}(t)=\frac{(k-1) !}{(i t)^{k-1}} \sum_{\ell=1}^{r} e^{i t v_{\ell}} \sum_{j=1}^{m_{\ell}} \frac{w_{\ell j}}{(j-1) !}(i t)^{j-1} .
$$

We will say that a random variable of density function $f_{D}(x)$ is a Vandermonde random variable of parameter $D(x)$.

Proposition 8 Let $D$ be a monic polynomial with at least 2 distinct roots and assume that all roots are nonnegative real. Then, the map $f_{D}$ is a density function having characteristic function $\Phi_{D}(t)$.

Proof. We first show that $\Phi_{D}(t)=\int_{-\infty}^{+\infty} f_{D}(x) e^{i t x} d x$. Set $I(t)=\int_{-\infty}^{\infty} f_{D}(x) e^{i t x} d x$ and observe that

$$
I(t)=(k-1) \sum_{h=2}^{r} \sum_{\ell=h}^{r} \sum_{j=1}^{m_{\ell}}\left(\begin{array}{l}
k-2 \\
j-1
\end{array}\right) w_{\ell j} \int_{v_{\ell-1}}^{v_{\ell}}\left(v_{\ell}-x\right)^{k-j-1} e^{i t x} d x .
$$

Integrating by parts one can verify that for $t \neq 0$ the function $e^{i t x}(c-x)^{p}$ admits the antiderivative

$$
\frac{e^{i t x}}{i t} \sum_{s=0}^{p} \frac{p !(c-x)^{p-s}}{(p-s) !(i t)^{s}}
$$

Hence we can write $I(t)=\sum_{h=2}^{r} \sum_{\ell=h}^{r}\left(A_{\ell, h}-A_{\ell, h-1}\right)$ where

$$
A_{\ell, h}=e^{i t v_{h}} \sum_{j=1}^{m_{\ell}} \frac{(k-1) !}{(j-1) !} w_{\ell j} \sum_{s=0}^{k-j-1} \frac{\left(v_{\ell}-v_{h}\right)^{k-j-1-s}}{(k-j-1-s) !(i t)^{s+1}}
$$


and in particular

$$
A_{\ell, \ell}=e^{i t v_{\ell}} \sum_{j=1}^{m_{\ell}} \frac{(k-1) !}{(j-1) !} \cdot \frac{w_{\ell j}}{(i t)^{k-j}} .
$$

Now, set $B_{h}=\sum_{\ell=h}^{r} A_{\ell, h}$ and $C_{h}=\sum_{\ell=h}^{r} A_{\ell, h-1}$. For each $2 \leq h \leq r-1$ we have $B_{h}-C_{h+1}=A_{h, h}$ and moreover $B_{r}=A_{r, r}$. Finally, reasoning as in Proposition 1 one can prove that $C_{2}=\sum_{\ell=1}^{r} A_{\ell, 1}-A_{1,1}=-A_{1,1}$. As a consequence, the integral can be computed as follows

$\int_{-\infty}^{\infty} f_{D}(x) e^{i t x} d x=\sum_{h=2}^{r}\left(B_{h}-C_{h}\right)=\sum_{\ell=1}^{r} A_{\ell, \ell}=\sum_{\ell=1}^{r} e^{i t v_{\ell}} \sum_{j=1}^{m_{\ell}} \frac{(k-1) !}{(j-1) !} \cdot \frac{w_{\ell j}}{(i t)^{k-j}}=\Phi_{D}(t)$.

The proposition is then proved if we show that $\lim _{t \rightarrow 0} \Phi_{D}(t)=1$. By expanding $e^{i t v \ell}$, we get

$$
\begin{aligned}
\Phi_{D}(t) & =\frac{(k-1) !}{(i t)^{k-1}} \sum_{\ell=1}^{r}\left(\sum_{j=0}^{\infty} \frac{v_{\ell}^{j}}{j !}(i t)^{j}\right) \cdot\left(\sum_{j=1}^{m_{\ell}} \frac{w_{\ell j}}{(j-1) !}(i t)^{j-1}\right) \\
& =(k-1) ! \sum_{s=1}^{\infty}\left(\sum_{\ell=1}^{r} \sum_{j=1}^{\min \left(s, m_{\ell}\right)} \frac{v_{\ell}^{s-j}}{(s-j) !} \cdot \frac{w_{\ell j}}{(j-1) !}\right)(i t)^{s-k} .
\end{aligned}
$$

By Proposition 1, the first non-null coefficient in the previous sum is obtained for $s=k$ and equals $1 /(k-1)$ !. This concludes the proof.

\section{$5 \quad$ Rational models for pattern statistics}

We now turn our attention to pattern statistics. Here, we recall the definition and the main properties of the rational stochastic models introduced in [2], based on the classical notion of rational formal series $[19,1]$.

Let $\mathbb{R}_{+}$be the semiring of nonnegative real numbers and consider the finite alphabet $\Sigma$. A formal series over $\Sigma$ with coefficients in $\mathbb{R}_{+}$is a function $r$ : $\Sigma^{*} \longrightarrow \mathbb{R}_{+}$, usually represented in the form $r=\sum_{\omega \in \Sigma^{*}}(r, \omega) \cdot \omega$, where $(r, \omega)$ denotes the value of $r$ at $\omega \in \Sigma^{*}$. Moreover, $r$ is called rational if it admits a linear representation, that is a triple $(\xi, \mu, \eta)$ where, for some integer $m>0, \xi$ and $\eta$ are (column) vectors in $\mathbb{R}_{+}^{m}$ and $\mu: \Sigma^{*} \longrightarrow \mathbb{R}_{+}^{m \times m}$ is a monoid morphism, such that $(r, \omega)=\xi^{T} \mu(\omega) \eta$ holds for each $\omega \in \Sigma^{*}$. Observe that considering such a triple $(\xi, \mu, \eta)$ is equivalent to defining a (weighted) nondeterministic automaton, where the set of states is given by $\{1,2, \ldots, m\}$ and the transitions, the initial and the final states are assigned weights in $\mathbb{R}_{+}$by $\mu, \xi$ and $\eta$, respectively. To avoid redundancy it is convenient to assume that $(\xi, \mu, \eta)$ is 
trim (meaning that all indices are used to define the series), i.e. for every index $i$ there are two indices $p, q$ and two words $x, y \in \Sigma^{*}$ such that $\xi_{p} \mu(x)_{p i} \neq 0$ and $\mu(y)_{i q} \eta_{q} \neq 0$. We say that $(\xi, \mu, \eta)$ is primitive if $\mathcal{M}=\sum_{\sigma \in \Sigma} \mu(\sigma)$ is a primitive matrix, that is for some $n \in \mathbb{N}$ all entries of $\mathcal{M}^{n}$ are strictly positive. We also recall that a matrix $\mathcal{M} \in \mathbb{R}_{+}^{m \times m}$ is called irreducible if for every pair of indices $p, q$ there exists $n \in \mathbb{N}$ such that $\mathcal{M}_{p q}^{n}>0$.

Any formal series can define a stochastic model for studying the frequency of occurrences of a letter in a word of given length. Consider the binary alphabet $\{a, b\}$ and, for any $n \in \mathbb{N}$, let $\{a, b\}^{n}$ denote the set of all words of length $n$ in $\{a, b\}^{*}$. Consider a formal series $r:\{a, b\}^{*} \longrightarrow \mathbb{R}_{+}$and let $n$ be a positive integer such that $(r, x) \neq 0$ for some $x \in\{a, b\}^{n}$. A probability measure over $\{a, b\}^{n}$ can be defined by setting

$$
\operatorname{Pr}\{\omega\}=\frac{(r, \omega)}{\sum_{x \in\{a, b\}^{n}}(r, x)} \quad\left(\omega \in\{a, b\}^{n}\right) .
$$

In particular, if $r$ is the characteristic series $\chi_{L}$ of a language $L \subseteq\{a, b\}^{*}$, then $\operatorname{Pr}$ is just the uniform probability function over $L \cap\{a, b\}^{n}$. Then, we define the random variable (r.v. for short) $Y_{n}:\{a, b\}^{n} \rightarrow\{0,1, \ldots, n\}$ such that $Y_{n}(\omega)=|\omega|_{a}$ for every $\omega \in\{a, b\}^{n}$. For every $j=0,1, \ldots, n$, we have

$$
\operatorname{Pr}\left\{Y_{n}=j\right\}=\frac{\sum_{|\omega|=n,|\omega|_{a}=j}(r, \omega)}{\sum_{x \in\{a, b\}^{n}}(r, x)} .
$$

If $r=\chi_{L}$ for some $L \subseteq\{a, b\}^{*}$, then $Y_{n}$ represents the number of occurrences of $a$ in a word chosen at random in $L \cap\{a, b\}^{n}$ under uniform distribution. We observe that, in this case, our results concerning $Y_{n}$ are related to the analysis of additive functions over strings [9].

When $r$ is rational, the r.v. $Y_{n}$ defines a model for the study of pattern statistics we call rational stochastic model. This is extention of the traditional Markovian models in the following sense. Given a regular set of patterns on an arbitrary finite alphabet $\Sigma$ consider a Markovian source $P$ generating words at random over $\Sigma$ and let $X_{n}$ be the r.v. representing the number of occurrences of patterns in a word of length $n$ generated by $P$; then there exists a rational formal series $r:\{a, b\}^{*} \longrightarrow \mathbb{R}_{+}$such that for every $n \geq 1$ the corresponding r.v. $Y_{n}$ has the same distribution as $X_{n}$ [2, Section 2.1].

Let $(\xi, \mu, \eta)$ be a linear representation for the rational series $r$ and set $\mathcal{A}=$ $\mu(a), \mathcal{B}=\mu(b), \mathcal{M}=\mathcal{A}+\mathcal{B}$. To study the behaviour of the random variables $Y_{n}$ and in particular their limit distribution, it is useful to introduce the sequence of functions $\left\{r_{n}(z)\right\}_{n}$ in the complex variable $z$ defined by

$$
r_{n}(z)=\sum_{x \in\{a, b\}^{n}}(r, x) \cdot e^{z|x|_{a}}=\xi^{T}\left(\mathcal{A} e^{z}+\mathcal{B}\right)^{n} \eta
$$

Indeed, it is immediate to see that the characteristic function of $Y_{n}$ satisfies 
the relation

$$
\Psi_{Y_{n}}(t)=\mathbb{E}\left(e^{i t Y_{n}}\right)=\frac{r_{n}(i t)}{r_{n}(0)}
$$

for $t \in \mathbb{R}$. We recall that a sequence of random variables $X_{n}$ converges in distribution to a random variable $X$ if and only if the sequence of characteristic functions $\Psi_{X_{n}}(t)$ pointwise converges to $\Psi_{X}(t)[7]$.

Now, consider the generating function of $\left\{r_{n}(z)\right\}_{n}$ and observe that

$$
\sum_{n=0}^{\infty} r_{n}(z) w^{n}=\xi^{T} H(z, w) \eta
$$

where $H(z, w)$ is the matrix defined by

$$
H(z, w)=\sum_{n=0}^{\infty}\left(\mathcal{A} e^{z}+\mathcal{B}\right)^{n} w^{n}=\left(I-w\left(\mathcal{A} e^{z}+\mathcal{B}\right)\right)^{-1}
$$

If $\mathcal{M}$ is irreducible, by Perron-Frobenius Theorem (see [21, Theorem 1.5]) it has a nonnegative real eigenvalue $\lambda$ of maximum modulus. Moreover, we know that the equation $\operatorname{Det}\left(y I-\mathcal{A} e^{z}-\mathcal{B}\right)=0$ defines an implicit function $y=y(z)$ which is analytic in a neighbourhood of $z=0$ and such that $y(0)=\lambda$.

If further $\mathcal{M}$ is primitive and $\mathcal{A} \neq 0 \neq \mathcal{B}$, then there are two constants $\beta \in(0,1), \gamma>0$, both depending on the matrix $\mathcal{M}$ and its eigenvectors (see [2] for details), such that, as $n$ tends to infinity, the following relations hold:

$$
\mathbb{E}\left(Y_{n}\right)=\beta n+\mathrm{O}(1), \quad \mathbb{V} a r\left(Y_{n}\right)=\gamma n+\mathrm{O}(1) .
$$

Finally, under the same hypothesis, one can prove that the distribution of $\frac{Y_{n}-\beta n}{\sqrt{\gamma n}}$ converges to the normal distribution of mean value 0 and variance 1 [2].

In our investigation we often deal with matrices of functions. We will say that a matrix $A(w)$ is a matrix function if all its entries are functions of the variable $w$. We will also say that $A(w)$ is analytic at a point $w=a$ if all its entries are analytic at the same point; moreover, its radius of convergence at that point is the smallest radius of convergence of the power series development of its entries (with center in $a$ ).

\subsection{Decomposition of a rational model}

Up to now, the properties of $Y_{n}$ have been studied only in the primitive models [2] and in the case of two primitive components [5]. Here, we present a general approach to deal with an arbitrary rational model. To this aim, we describe the construction of the reduced graph of the strongly connected components of the corresponding linear representation. This is a usual approach in the 
analysis of counting problems on regular languages (see for instance [8] for an application concerning trace languages).

Let $(\xi, \mu, \eta)$ be a linear representation over the alphabet $\{a, b\}$ with coefficients in $\mathbb{R}_{+}$. As in the previous section, set $\mathcal{A}=\mu(a), \mathcal{B}=\mu(b), \mathcal{M}=\mathcal{A}+\mathcal{B}$ and consider the directed graph defined by $\mathcal{M}$, where the set of nodes is $\{1,2, \ldots, m\}$ and $(p, q)$ is an (oriented) edge if and only if $\mathcal{M}_{p q} \neq 0$. Then, let $C_{1}, C_{2}, \ldots, C_{s}$ be the strongly connected components of the graph and define $C_{i}$ initial (resp. final) if $\xi_{p} \neq 0$ (resp. $\eta_{p} \neq 0$ ) for some $p \in C_{i}$. The reduced graph of $(\xi, \mu, \eta)$ is then defined as the directed acyclic graph $G$ where $C_{1}, C_{2}, \ldots, C_{s}$ are the vertices and any pair $\left(C_{i}, C_{j}\right)$ is an edge if and only if $i \neq j$ and $\mathcal{M}_{p q} \neq 0$ for some $p \in C_{i}$ and some $q \in C_{j}$.

Up to a permutation of indices, the matrix $\mathcal{M}$ can be represented as a triangular block matrix of the form

$$
\mathcal{M}=\left(\begin{array}{ccccc}
M_{1} & M_{12} & M_{13} & \cdots & M_{1 s} \\
0 & M_{2} & M_{23} & \cdots & M_{2 s} \\
& & \cdots & & \\
0 & 0 & 0 & \cdots & M_{s}
\end{array}\right)
$$

where each $M_{i}$ corresponds to the strongly connected component $C_{i}$ and every $M_{i j}$ corresponds to the transitions from vertices of $C_{i}$ to vertices of $C_{j}$ in the original graph of $\mathcal{M}$. Also $\mathcal{A}, \mathcal{B}, \xi$ and $\eta$ admit similar decompositions: we define the matrices $A_{i}, A_{i j}, B_{i}, B_{i j}$ and the vectors $\xi_{i}, \eta_{i}$ in the corresponding way and we say that the component $C_{i}$ is degenerate if $A_{i}=0$ or $B_{i}=0$. Since each matrix $M_{i}$ is either null or irreducible, by Perron-Frobenius Theorem it has a nonnegative real eigenvalue $\lambda_{i}$ of maximum modulus. We call main eigenvalue of $\mathcal{M}$ the value $\lambda=\max \left\{\lambda_{i} \mid i=1,2, \ldots, s\right\}$ and we say that $C_{i}$ is a dominant component if $\lambda_{i}=\lambda$. Observe that $\lambda_{i}=0$ only if $C_{i}$ reduces to a loopless single node and hence from now on we assume $\lambda>0$.

Further, if a matrix $M_{i}$ is primitive, we say that $C_{i}$ is a primitive component. In this case, when $C_{i}$ is not degenerate (i.e. $A_{i} \neq 0 \neq B_{i}$ ), we may consider the constants $\beta_{i}$ and $\gamma_{i}$ associated with $M_{i}$ defined as in (15); we have $0<\beta_{i}<1$ and $\gamma_{i}>0$. On the contrary, if $C_{i}$ is degenerate, it is natural to set $\gamma_{i}=0$ and define $\beta_{i}=0$ or $\beta_{i}=1$ according whether $A_{i}=0$ or $B_{i}=0$ (so that (15) still holds true for a degenerate r.v.). Thus the constants $\beta_{i}$ and $\gamma_{i}$ are well-defined for every primitive component $C_{i}$ : we say they are the mean constant and the variance constant of $C_{i}$, respectively.

The block decomposition of $\mathcal{M}$ also induces a decomposition of the matrix $H(z, w)$ defined in (14). More precisely, the blocks under the diagonal are all null, while the upper triangular part is composed by a family of matrices, say $H_{i j}(z, w), 1 \leq i \leq j \leq s$. Note that the bivariate generating function 
$\xi^{T} H(z, w) \eta$, which is the main tool of our investigation, is now given by

$$
\xi^{T} H(z, w) \eta=\sum_{n=0}^{\infty} \xi^{T}\left(\mathcal{A} e^{z}+\mathcal{B}\right)^{n} \eta \cdot w^{n}=\sum_{1 \leq i \leq j \leq s} \xi_{i}^{T} H_{i j}(z, w) \eta_{j} .
$$

Setting $M_{i j}(z)=A_{i j} e^{z}+B_{i j}$ and reasoning by induction on $j-i$, one can prove that, for each $1 \leq i \leq j \leq s$,

$$
H_{i j}(z, w)= \begin{cases}\left(I-w\left(A_{i} e^{z}+B_{i}\right)\right)^{-1} & \text { if } j=i \\ \sum_{*} H_{i_{1} i_{1}}(z, w) M_{i_{1} i_{2}}(z) H_{i_{2} i_{2}}(z, w) \cdots M_{i_{\ell-1} i_{\ell}}(z) H_{i_{\ell} i_{\ell}}(z, w) \cdot w^{\ell-1} & \text { if } j \neq i\end{cases}
$$

where the $\operatorname{sum}(*)$ is extended over all sequences of integers $\left(i_{1}, i_{2}, \ldots, i_{\ell}\right)$, $\ell \geq 2$ such that $i_{1}=i, i_{t}<i_{t+1}$ for each $t=1, \ldots, \ell-1$ and $i_{\ell}=j$.

Equation (18) suggests us to introduce the notion of chain of the reduced graph $G$ associated with $(\xi, \mu, \eta)$. A chain is a simple path in $G$, i.e. a sequence of distinct components $\kappa=\left(C_{i_{1}}, C_{i_{2}}, \ldots, C_{i_{\ell}}\right)$ where $\ell \geq 1$, such that $M_{i_{j} i_{j+1}} \neq 0$ for every $j=1,2, \ldots, \ell-1$. We say that $\ell$ is the length of $\kappa$ while the order of $\kappa$ is the number of its dominant components. We also denote by $\Gamma$ the family of all chains in $G$ starting with an initial component and ending with a final component. Note that, the linear representation $(\xi, \mu, \eta)$ being trim, each component lies over at least one chain in $\Gamma$. We say that a chain $\kappa$ is a main chain if $\kappa \in \Gamma$ and its order is maximal in $\Gamma$. We denote by $\Gamma_{m}$ the set of all main chains in $G$.

Example 1 Here, we present a parametric example depending on two constants $\alpha \in \mathbb{R}$ and $\rho \in[0,1]$. For such parameters let us consider the formal series $s_{\alpha, \rho}$ having linear representation $(\pi, \nu, \tau)$ such that

$$
\pi^{\prime}=(1,0), \quad \nu_{\alpha, \rho}(a)=\alpha \cdot\left(\begin{array}{ll}
0 & 1 \\
\rho & 0
\end{array}\right), \quad \nu_{\alpha, \rho}(b)=\alpha \cdot\left(\begin{array}{rr}
1 & 0 \\
1-\rho & 0
\end{array}\right), \quad \tau=\left(\begin{array}{l}
1 \\
0
\end{array}\right) .
$$

We note that the Perron-Frobenius eigenvalue associated with this linear representation is $\alpha(1+\sqrt{5}) / 2$.

Given two families of parameters $\left\{\alpha_{i}\right\}_{i=1, m}$ and $\left\{\rho_{i}\right\}_{i=1, m}$, let us define the formal series $r$ given by the Cauchy product $r=\prod_{i=1}^{m} s_{\alpha_{i}}, \rho_{i}$. Its linear representation of size $2 m$ is given by $(\xi, \mu, \eta)$ where $\xi_{1}=1$ and $\xi_{i}=0$ for every $i \neq 1, \eta_{2 i}=0$ and $\eta_{2 i+1}=1$ for every $i$, while for $x \in\{a, b\} \mu$ is defined by

$$
\mu(x)=\left(\begin{array}{ccccc}
\nu_{\alpha_{1}, \rho_{1}}(x) & M_{12}(x) & M_{13}(x) & \cdots & M_{1 m}(x) \\
0 & \nu_{\alpha_{2}, \rho_{2}}(x) & M_{23}(x) & \cdots & M_{2 m}(x) \\
0 & \cdots & & \\
0 & 0 & 0 & \cdots & \nu_{\alpha_{m}, \rho_{m}}(x)
\end{array}\right)
$$


with

$$
M_{i j}(a)=\left(\begin{array}{cc}
\alpha_{j} & 0 \\
0 & 0
\end{array}\right) \quad \text { and } \quad M_{i j}(b)=\left(\begin{array}{cc}
0 & \alpha_{j} \\
0 & 0
\end{array}\right)
$$

Here, the entries $1,2, \cdots, 2 m$ can be gathered in strongly connected components, defined by the sets $C_{i}=\{2 i-1,2 i\}$ for $i=1,2, \cdots, m$. Thus, the reduced graph of $(\xi, \mu, \eta)$ consists of nodes $C_{i}$ 's and edges $\left(C_{i}, C_{j}\right)$ with $i<j$. The component $C_{1}$ is initial while all $C_{j}$ 's are final. The orders of the chains depend on the values $\alpha_{i}$ 's. In particular, when all $\alpha_{i}$ 's are equal, we have only one main chain of order $m$. If the $\alpha_{i}$ 's are different, there may be several main chains. For instance, if $m=4, \alpha_{2}=\alpha_{3}$ and $\alpha_{1}=\alpha_{4}=2 \alpha_{2}$, only $C_{1}$ and $C_{4}$ are dominant; therefore we have four main chains, namely $\left(C_{1}, C_{2}, C_{3}, C_{4}\right)$, $\left(C_{1}, C_{2}, C_{4}\right),\left(C_{1}, C_{3}, C_{4}\right)$ and $\left(C_{1}, C_{4}\right)$.

\subsection{The role of main chains}

In this section we study the properties of main chains and in particular we show that they determine the limit distribution of the sequence $\left\{Y_{n}\right\}$ associated with the linear representation $(\xi, \mu, \eta)$. Intuitively, this is a consequence of two facts. First, the characteristic function of (a normalization of) $Y_{n}$ depends on the sequences $\left\{r_{n}(z)\right\}$ for $z$ near 0 , and hence on the generating function $\xi^{T} H(z, w) \eta$. Second, by (17), this function is a sum of products of the form given in (18), each of which is identified by a chain: the products corresponding to the main chains have singularities of smallest modulus with the largest degree, and hence they yield the main asymptotic contribution to the associated sequence $\left\{r_{n}(z)\right\}$.

So, let us take in exam the terms of the sum in the right hand side of (17). First we consider the case $i=j$ and, for every $j=1,2, \ldots, s$, we denote $\left\{r_{n}^{(j)}(z)\right\}$ the sequence given by

$$
\xi_{j}^{T} H_{j j}(z, w) \eta_{j}=\sum_{n=0}^{\infty} r_{n}^{(j)}(z) w^{n}
$$

By relation (18), we have

$$
H_{j j}(z, w)=\left(I-w\left(A_{j} e^{z}+B_{j}\right)\right)^{-1}=\frac{\operatorname{Adj}\left(I-w\left(A_{j} e^{z}+B_{j}\right)\right)}{\operatorname{det}\left(I-w\left(A_{j} e^{z}+B_{j}\right)\right)}
$$

and hence, as $z$ tends to 0 , the singularities of each entry approach the inverses of eigenvalues of $M_{j}$. We can distinguish three cases according to the properties of $M_{j}$ :

i) $M_{j}$ is primitive and dominant. Then, $\lambda$ is its (sole) eigenvalue of largest modulus. The equation $\operatorname{det}\left(y I-\left(A_{j} e^{z}+B_{j}\right)\right)=0$ implicitly defines a function $y=y_{j}(z)$ in a neighbourhood of $z=0$ such that $y_{j}(0)=\lambda$. Such a 
function is analytic at the point $z=0$ and admits an expansion of the form

$$
y_{j}(z)=\lambda\left(1+\beta_{j} z+\frac{\gamma_{j}+\beta_{j}^{2}}{2} z^{2}+\mathrm{O}\left(z^{3}\right)\right)
$$

where $\beta_{j}$ and $\gamma_{j}$ are the mean and variance constants of $C_{j}$. Note that this equation is well-defined also when $C_{i}$ is degenerate (in particular, if $A_{i}=0$ then $y_{i}(z)=\lambda$ for all $z$ ).

Then, there exists a matrix function $R_{j}(z)$ analytic and nonnull at $z=0$ such that, for every $z$ near 0 ,

$$
H_{j j}(z, w)-\frac{R_{j}(z)}{1-y_{j}(z) w}
$$

has a radius of convergence strictly greater than $\lambda^{-1}$. As a consequence we have

$$
r_{n}^{(j)}(z)=\xi_{j}^{T} R_{j}(z) \eta_{j}\left(y_{j}(z)\right)^{n}+\mathrm{O}\left(\rho^{n}\right)
$$

for some $0<\rho<\lambda$ and every $z$ near 0 .

ii) $M_{j}$ is dominant (but not necessarily primitive). Then, we can consider the family $E_{j}$ of the eigenvalues of $M_{j}$ of largest modulus. By PerronFrobenius Theorem, we know $\lambda \in E_{j}$ and for every $\alpha \in E_{j}$ the equation $\operatorname{det}\left(y I-\left(A_{j} e^{z}+B_{j}\right)\right)=0$ implicitly defines a function $y=y_{\alpha}(z)$ in a neighbourhood of $z=0$ such that $y_{\alpha}(0)=\alpha$. Also $y_{\alpha}(z)$ is analytic at $z=0$, where it admits an expansion of the form

$$
y_{\alpha}(z)=\alpha\left(1+m_{\alpha} z+s_{\alpha} z^{2}+\mathrm{O}\left(z^{3}\right)\right)
$$

with $m_{\alpha} \in \mathbb{R}_{+}$and $\Re\left(s_{\alpha}\right) \geq 2 m_{\alpha}^{2}$ (consequence of point (e) in [21, Theo.1.5]). Reasoning as above this implies, for $z$ near 0 and some $0<\rho<\lambda$,

$$
r_{n}^{(j)}(z)=\sum_{\alpha \in E_{j}} \xi_{j}^{T} R_{\alpha}(z) \eta_{j}\left(y_{\alpha}(z)\right)^{n}+\mathrm{O}\left(\rho^{n}\right)
$$

where $R_{\alpha}(z)$ is a matrix function analytic and nonnull at $z=0$, for each $\alpha \in E_{j}$.

iii) $M_{j}$ is not dominant. Then, all its eigenvalues are in modulus smaller than $\lambda$ and hence, as $z$ is near to 0 the radius of convergence of $H_{j j}(z, w)$ is greater than $\lambda^{-1}$. This implies $r_{n}^{(j)}(z)=\mathrm{O}\left(\rho^{n}\right)$ for some $0<\rho<\lambda$ and all $z$ near 0 .

Now, let us study the behaviour of $H_{i j}(z, w)$ for $i \neq j$. Recalling (18), we consider an arbitrary chain $\kappa=\left(C_{i_{1}}, C_{i_{2}}, \ldots, C_{i_{\ell}}\right)$ with $\ell \geq 2$ and we denote by $H_{\kappa}(z, w)$ the corresponding matrix given by

$$
H_{\kappa}(z, w)=H_{i_{1} i_{1}}(z, w) M_{i_{1} i_{2}}(z) H_{i_{2} i_{2}}(z, w) \cdots M_{i_{\ell-1} i_{\ell}}(z) H_{i_{\ell} i_{\ell}}(z, w) \cdot w^{\ell-1}
$$

We also define the sequence $\left\{r_{n}^{(\kappa)}(z)\right\}$ by

$$
\xi_{i_{1}}^{T} H_{\kappa}(z, w) \eta_{i_{\ell}}=\sum_{n=0}^{\infty} r_{n}^{(\kappa)}(z) w^{n} .
$$


Then, the next proposition can be proved by applying the previous properties to $(22)$.

Proposition 9 Let $\kappa$ be a chain in $\Gamma$ of order $k \geq 0$. Then, as $n$ tends to $+\infty$, the following statements hold for every $c \in \mathbb{C}$ and every $t \in \mathbb{R}$ :

(1) If $k=0$ then $r_{n}{ }^{(\kappa)}(c / n)=O\left(\rho^{n}\right)$ for some $0<\rho<\lambda$;

(2) If $k \geq 1$ then $r_{n}{ }^{(\kappa)}(c / n)=O\left(\lambda^{n} n^{k-1}\right)$;

(3) If $k \geq 1$ and the dominant components of $\kappa$ are primitive, then $r_{n}{ }^{(\kappa)}(c / n)=$ $\Theta\left(\lambda^{n} n^{k-1}\right)\left({ }^{2}\right)$

(4) If $k \geq 1$ then $r_{n}{ }^{(\kappa)}(i t / \sqrt{n})=O\left(\lambda^{n} n^{k-1}\right)$.

Proof. Without loss of generality, we may assume $\kappa=\left(C_{1}, C_{2}, \ldots, C_{\ell}\right)$. Then, we have

$$
H_{\kappa}(z, w)=H_{11}(z, w) M_{12}(z) H_{22}(z, w) \cdots M_{\ell-1 \ell}(z) H_{\ell \ell}(z, w) \cdot w^{\ell-1}
$$

and it is clear that, for any fixed $z$, the singularities of $\xi_{1}^{T} H_{\kappa}(z, w) \eta_{\ell}$ are those of the matrices $H_{j j}(z, w)$ for $j=1,2, \ldots, \ell$. If $k=0$ and $z$ near 0 , the radius of convergence of each $H_{j j}(z, w)$ is greater than $\lambda^{-1}$ and hence $r_{n}{ }^{(\kappa)}(z)=\mathrm{O}\left(\rho^{n}\right)$ for some $0<\rho<\lambda$, which proves point 1 .

Now, set $I=\left\{j: C_{j}\right.$ is dominant $\}$ and assume $k=\sharp I \geq 1$. Let $j \in I$ and let $z$ be a complex value near 0 . By property ii), the dominant singularities of $H_{j j}(z, w)$ are the simple poles $y_{\alpha}(z)^{-1}$, where $\alpha \in E_{j}$. Thus, the same values are poles for $\xi_{1}^{T} H_{\kappa}(z, w) \eta_{\ell}$ of degree $k$ at most. Hence, $r_{n}{ }^{(\kappa)}(z)$ is bounded by a linear combinations of terms of the form $\mathrm{O}\left(y_{\alpha}(z)^{n} n^{k-1}\right)$, where $\alpha \in \bigcup_{j \in I} E_{j}$; setting $z=c / n$, by (21), each of them is of the order $\mathrm{O}\left(\lambda^{n} n^{k-1}\right)$, which proves point 2 .

Analogously, setting $z=i t n^{-1 / 2}$, again by (21) for every $\alpha$ we have

$$
\left|y_{\alpha}\left(i t n^{-1 / 2}\right)^{n}\right|=\left|\alpha^{n}\left(1+m_{\alpha} \frac{i t}{\sqrt{n}}+\mathrm{O}(1 / n)\right)\right|^{n} ;
$$

since $m_{\alpha} \in \mathbb{R}$ this implies

$$
\left|y_{\alpha}\left(i t n^{-1 / 2}\right)^{n}\right|=\lambda^{n}\left(1+\frac{m_{\alpha}^{2} t^{2}}{n}\right)^{n / 2} \mathrm{O}(1+1 / n)=\mathrm{O}\left(\lambda^{n}\right)
$$

and hence $r_{n}{ }^{(\kappa)}(i t / \sqrt{n})=\mathrm{O}\left(\sum_{\alpha} y_{\alpha}\left(i t n^{-1 / 2}\right)^{n} n^{k-1}\right)=\mathrm{O}\left(\lambda^{n} n^{k-1}\right)$, which proves point 4

Finally, assume $C_{j}$ primitive for every $j \in I$ and let $z$ be a complex value near 0 . Then, the main singularities of $\xi_{1}^{T} H_{\kappa}(z, w) \eta_{\ell}$ are the values $y_{j}(z)^{-1}$ defined

$\overline{2}$ In this work, for any pair of sequences $\left\{f_{n}\right\},\left\{g_{n}\right\} \subseteq \mathbb{C}$, the expression $f_{n}=\Theta\left(g_{n}\right)$ means that for two positive constants $a, b$ the relation $a\left|g_{n}\right| \leq\left|f_{n}\right| \leq b\left|g_{n}\right|$ holds for every $n$ large enough. 
in i). By (24) this implies

$$
\xi_{1}^{T} H_{\kappa}(z, w) \eta_{\ell}=\frac{R(z, w)}{\Pi_{j \in I}\left(1-y_{j}(z) w\right)}
$$

where $R(z, w)$ is a function analytic in a disk $\left\{w \in \mathbb{C}|| w \mid \leq \lambda^{-1}+d\right\}$, for some $d>0$. Thus, the leading term of $r_{n}{ }^{(\kappa)}(z)$ is determined by the convolution of the sequences $\left\{y_{j}(z)^{n}\right\}_{n}$, for $j \in I$; hence, setting $z=c / n$ and using (20) we get $r_{n}{ }^{(\kappa)}(c / n)=\Theta\left(\lambda^{n} n^{k-1}\right)$ proving point 3 .

Since by Eq. (17), we have $r_{n}(z)=\sum_{\kappa \in \Gamma} r_{n}^{(\kappa)}(z)$, we obtain the following result, which shows the key role of the main chains. Also note that the property does not hold if the main chains admit non-primitive dominant components.

Theorem 10 If all dominant components of the main chains are primitive then, for every constant $c \in \mathbb{C}$, we have

$$
r_{n}(c / n)=\sum_{\kappa \in \Gamma_{m}} r_{n}^{(\kappa)}(c / n)(1+O(1 / n))=\Theta\left(\lambda^{n} n^{k-1}\right)
$$

where $k$ is the order of the main chains.

\section{Limit distributions in multicomponent models}

Theorem 10 shows that in a multicomponent model the asymptotic behaviour of our statistics mainly depends on the main chains. This fact leads to study the relevant case when the model has just one main chain. In this case, assuming further mild conditions on the dominant components, it turns out that the limit distribution of $Y_{n} / n$ coincides with a Vandermonde distribution. For this reason we introduce the notion of simple model.

Let $(\xi, \mu, \eta)$ be a linear representation over the alphabet $\{a, b\}$ with coefficients in $\mathbb{R}_{+}$. We say that $(\xi, \mu, \eta)$ is a simple linear representation, or just a simple model, if $\Gamma_{m}$ contains only one chain $\kappa$ and every dominant component in $\kappa$ is primitive.

In simple models the limit distribution of $Y_{n}$ first depends on the order $k$ of $\kappa$, i.e. the number of its dominant components. If $k \leq 2$ the limit distribution is known and derives from the analysis of the bicomponent models given in [5]; in particular (if the dominant components are not degenerate) we have the following results:

- If $\kappa$ has only one dominant component $C_{i}$ then the limit distribution of $\frac{Y_{n}-\beta_{i} n}{\sqrt{\gamma_{i} n}}$ is a Gaussian distribution of mean value 0 and variance 1; 
- If $\kappa$ has two dominant components $C_{i}, C_{j}$ then we have the following three subcases:

(1) If $\beta_{i} \neq \beta_{j}$ then $Y_{n} / n$ converges in law to a random variable uniformly distributed in the interval $\left[b_{1}, b_{2}\right]$, where $b_{1}=\min \left\{\beta_{i}, \beta_{j}\right\}$ and $b_{2}=\max \left\{\beta_{i}, \beta_{j}\right\}$;

(2) If $\beta_{i}=\beta_{j}=\beta$ but $\gamma_{i} \neq \gamma_{j}$ then the limit distribution of $\frac{Y_{n}-\beta n}{\sqrt{n}}$ is a mixture of normal distributions of mean value 0 and variance uniformly distributed in the interval $\left[c_{1}, c_{2}\right]$, where $c_{1}=\min \left\{\gamma_{i}, \gamma_{j}\right\}$ and $c_{2}=\max \left\{\gamma_{i}, \gamma_{j}\right\}$. In other words, $\frac{Y_{n}-\beta n}{\sqrt{n}}$ converges in law to a random variable with density function

$$
f(x)=\frac{1}{c_{2}-c_{1}} \int_{c_{1}}^{c_{2}} \frac{e^{-x^{2} /(2 v)}}{\sqrt{2 \pi v}} d v
$$

which has characteristic function

$$
F(t)=2 \frac{e^{-\frac{c_{1} t^{2}}{2}}-e^{-\frac{c_{2} t^{2}}{2}}}{\left(c_{2}-c_{1}\right) t^{2}} .
$$

Notice that $F(t)=\Phi_{P}\left(i t^{2} / 2\right)$ where $P(x)=\left(x-c_{1}\right)\left(x-c_{2}\right)$;

(3) If $\beta_{i}=\beta_{j}=\beta$ and $\gamma_{i}=\gamma_{j}=\gamma$ then the distribution of $\frac{Y_{n}-\beta n}{\sqrt{\gamma n}}$ again converges to a Gaussian distribution of mean value 0 and variance 1 .

Here, we determine the limit distribution of $Y_{n} / n$ for simple models having main chain $\kappa$ of order $k \geq 2$. We only assume that $\kappa$ has at least two dominant components with different mean constants. In Section 7 we extend this result to the case when all dominant components of $\kappa$ have the same mean constant.

Theorem 11 Let $Y_{n}$ be defined in a simple model with main chain $\kappa$ of order $k \geq 2$. Let $\beta_{1}, \ldots, \beta_{r}$ denote the mean constants of the dominant components in $\kappa$ in increasing order and assume $r \geq 2$. Also, for each $\ell=1,2, \ldots, r$, let $m_{\ell}$ be the multiplicity of $\beta_{\ell}$, that is the number of dominant components in $\kappa$ whose mean constant equals $\beta_{\ell}$. Then, $Y_{n} / n$ converges in law to a Vandermonde random variable associated with the polynomial $P(x)=\prod_{\ell=1}^{r}\left(x-\beta_{\ell}\right)^{m_{\ell}}$.

Observe that in the case $k=2$ we obtain the result stated in point (1) above. The proof of the theorem is based on the analysis of the characteristic function of $Y_{n} / n$, which by equation (13) is given by

$$
\Psi_{Y_{n} / n}(t)=\frac{r_{n}(i t / n)}{r_{n}(0)} .
$$

Thus, we first present the following lemma, which provides a useful expression for $r_{n}(i t / n)$. To this aim, as in Eq. (7), let $\left\{g_{Q}(n)\right\}_{n}$ be the sequence having generating function $Q(0) / Q(x)$, where

$$
Q(x)=\prod_{\ell=1}^{r}\left(x-\frac{1}{1+\beta_{\ell} i t / n}\right)^{m_{\ell}} .
$$


Lemma 12 Assume the hypotheses of Theorem 11. Then, for every $t \in \mathbb{R}$, as $n$ grows to $+\infty$ we have

$$
r_{n}\left(\frac{i t}{n}\right)=\sum_{s=0}^{k-1} \lambda^{n-s} a_{s}\left(\frac{i t}{n}\right) \cdot g_{Q}(n-s) \cdot(1+O(1 / n))
$$

and in particular

$$
r_{n}(0)=\frac{n^{k-1}}{(k-1) !}\left(\sum_{s=0}^{k-1} \lambda^{n-s} a_{s}(0)\right) \cdot(1+O(1 / n))
$$

where, for each $s, a_{s}(z)$ is an analytic function at $z=0$.

Proof. By Theorem 10, we get $r_{n}(i t / n)=r_{n}^{(\kappa)}(i t / n)(1+\mathrm{O}(1 / n))$ and hence we have to show that $r_{n}^{(\kappa)}(i t / n)$ equals the right hand side of $(27)$. We can evaluate $r_{n}^{(\kappa)}(i t / n)$ by refining the proof of point 3 in Proposition 9. Indeed, since $H_{\kappa}(z, w)$ satisfies equation $(25)$, we have

$$
\xi_{1}^{T} H_{\kappa}(z, w) \eta_{\ell}=\sum_{s=0}^{k-1} a_{s}(z) w^{s} \cdot \prod_{\ell=1}^{r}\left(1-f_{\ell}(z) w\right)^{-m_{\ell}}+G(z, w)
$$

where each $a_{s}(z)$ is a polynomial in $e^{z}, f_{\ell}(z)=\lambda\left(1+\beta_{\ell} z+O(z)\right)$ for every $\ell=1,2, \ldots, r$ and, for all $z$ near 0 , the function $G(z, w)$ is analytic in a disk $\left\{w \in \mathbb{C}|| w \mid \leq \lambda^{-1}+d\right\}$, for some $d>0$.

Clearly $\prod_{\ell=1}^{r}\left(1-f_{\ell}(z) w\right)^{-m_{\ell}}$ is the generating function of the sequence whose $n$-th term is

$$
\sum_{\sum_{\ell} n_{\ell}=n} \prod_{\ell=1}^{r}\left(\begin{array}{c}
n_{\ell}+m_{\ell}-1 \\
m_{\ell}-1
\end{array}\right) f_{\ell}(z)^{n_{\ell}} .
$$

Setting $z=i t / n$ and recalling equation (7), the previous expression can be re-written as $\lambda^{n} g_{Q}(n) \cdot(1+\mathrm{O}(1 / n))$. Thus, since (29) is the generating function of $\left\{r_{n}^{(\kappa)}(z)\right\}_{n}$, the main term of $r_{n}^{(\kappa)}(i t / n)$ is given by the convolution of $\left\{a_{n}(i t / n)\right\}_{n}$ and $\left\{\lambda^{n} g_{Q}(n)\right\}_{n}$, which leads to equation (27). Equation (28) follows by noting that if $t=0$, then $Q(x)=(x-1)^{k}$ and $g_{Q}(n)=\left(\begin{array}{c}n+k-1 \\ k-1\end{array}\right)$.

Proof of Theorem 11. As $n$ grows to infinity, the behaviour of $g_{Q}(n-s)$ does not depend on $s$. To prove this fact we need the following equalities, that can be proved from the definitions given in Section 3. Set $v_{\ell}=\left(1-\beta_{\ell} i t / n\right)^{-1}$, for every $\ell, j=1,2, \ldots r$ we have

$$
\begin{gathered}
\left(v_{\ell}\right)^{-n}=e^{-i t \beta_{\ell}} \cdot(1+\mathrm{O}(1 / n)) \\
\left(v_{\ell}-v_{j}\right)^{-1}=\left(-\frac{n}{i t}\right)\left(\beta_{\ell}-\beta_{j}\right)^{-1} \cdot(1+\mathrm{O}(1 / n)) .
\end{gathered}
$$


Moreover, if $w_{\ell j}$ and $c_{\ell j}$ are the entries of the last column of the Vandermonde matrices associated with the polynomial $Q(x)$ and $P(x)=\prod_{\ell=1}^{r}\left(x-\beta_{\ell}\right)^{m_{\ell}}$, respectively, then using Eq (6) one can obtain

$$
w_{\ell j}=\left(-\frac{n}{i t}\right) c_{\ell j} \cdot(1+\mathrm{O}(1 / n)) .
$$

As a consequence, applying Proposition 3 and using the previous equalities, one proves that

$$
g_{Q}(n-s)=\left(\frac{n}{i t}\right)^{k-1}\left(\sum_{\ell=1}^{r} e^{i t \beta_{\ell}} \sum_{j=1}^{m_{\ell}} c_{\ell j} \frac{(i t)^{j-1}}{(j-1) !}\right) \cdot(1+\mathrm{O}(1 / n)) .
$$

Replacing the previous expression into (27) we get

$r_{n}\left(\frac{i t}{n}\right)=\left(\frac{n}{i t}\right)^{k-1}\left(\sum_{\ell=1}^{r} e^{i t \beta_{\ell}} \sum_{j=1}^{m_{\ell}} c_{\ell j} \frac{(i t)^{j-1}}{(j-1) !}\right)\left(\sum_{s=0}^{k-1} \lambda^{n-s} a_{s}\left(\frac{i t}{n}\right)\right) \cdot(1+\mathrm{O}(1 / n))$.

Hence, applying equation (26) and recalling equation (28), one can see that the characteristic function $\Psi_{Y_{n} / n}(t)$ converges to $\Phi_{P}(t)$ for every $t \in \mathbb{R}$. This proves the result.

\section{$7 \quad$ Further results}

The analysis presented in the previous section can be extended to all simple models, also when the mean constants $\beta_{j}$ 's (associated with the dominant components of the main chain) are totally coincident. In this case, clearly $Y_{n}^{(\kappa)} / n$ converges in ditribution to such a constant, and it is natural to consider a finer normalization. With respect to this point, the following theorem holds, which can be proved as Theorem 11.

Theorem 13 Let $Y_{n}$ be defined in a simple model having main chain $\kappa$ of order $k \geq 2$ and assume that all dominant components in $\kappa$ have the same mean constant $\beta$. Let $\gamma_{1}, \ldots, \gamma_{s}$ be the distinct variance constants (in increasing order) of the dominant components in $\kappa$ and let $m_{1}, m_{2}, \ldots, m_{s}$ denote their multiciplities. If $s=1$ and $\gamma_{1} \neq 0$, then $\left(Y_{n}-\beta n\right) / \sqrt{\gamma_{1} n}$ converges in distribution to a normal random variable of mean 0 and variance 1 . Otherwise, if $s>1$, then $\left(Y_{n}-\beta n\right) / \sqrt{n}$ converges in distribution to a random variable having characteristic function $\Phi_{P}\left(i t^{2} / 2\right)$, where $\Phi_{P}(t)$ is the characteristic function of a Vandermonde random variable of parameter $P(x)=\prod_{\ell=1}^{s}\left(x-\gamma_{\ell}\right)^{m_{\ell}}$.

Notice that, for $k=2$, the previous theorem reduces to points (2) and (3) at page 21. 
The results of Theorem 11, concerning the limit distribution of $Y_{n}^{(\kappa)} / n$, can be further extended by a standard conditioning argument (already used in [5]) to all rational models $(\xi, \mu, \eta)$ whose main chains are "simple", i.e. for every $\kappa \in \Gamma_{m}$ all dominant components in $\kappa$ are primitive. In this case, by Eqs. (22) and (23), for every $\kappa \in \Gamma_{m}$ one can easily see that

$$
r_{n}^{(\kappa)}(z)=s_{\kappa}(z) \lambda^{n} n^{k-1}+\mathrm{O}\left(\lambda^{n} n^{k-2}\right)
$$

where $k$ is the degree of $\kappa$ and $s_{\kappa}(z)$ is a non-null analytic function at $z=0$. Then, by Theorem 10, we have

$$
r_{n}(0)=R \lambda^{n} n^{k-1}+\mathrm{O}\left(\lambda^{n} n^{k-2}\right)
$$

where $R=\sum_{\kappa \in \Gamma_{m}} s_{\kappa}(0)$. We can also associate each $\kappa \in \Gamma_{m}$ with the probability value $p_{\kappa}$, given by $p_{\kappa}=s_{\kappa}(0) / R$. Note that the values $\left\{p_{\kappa}\right\}_{\kappa \in \Gamma_{m}}$ define a discrete probability measure and they can be explicitly computed from the triple $(\xi, \mu, \eta)$.

Moreover, each $\kappa \in \Gamma_{m}$ defines a simple rational model in its own right, with an associate sequence of random variables $\left\{Y_{n}^{(\kappa)}\right\}$. The limit distribution of $Y_{n}^{(\kappa)} / n$ can be studied by applying Theorem 11 . In particular, $Y_{n}^{(\kappa)} / n$ always converges in distribution to a random variable of distribution function $F_{\kappa}(x)$ defined according to the previous results. Note that, if all constants $\beta_{j}$ 's are here equal, then $F_{\kappa}(x)$ reduces to the degenerate distribution of mass point $\beta_{1}$. Now, it is not difficult to see that the overall statistics $Y_{n} / n$ converges in distribution to a r.v. of distribution function $F(x)$ defined by $F(x)=\sum_{\kappa \in \Gamma_{m}} F_{\kappa}(x) p_{\kappa}$. This completes our analysis of the limit distribution of $Y_{n}^{(\kappa)} / n$. The only family of rational stochastic models not coverered by our results consists of those models having a main chain with some non-primitive dominant component; in those cases, periodicity phenomena occur.

\section{References}

[1] J. Berstel and C. Reutenauer. Rational series and their languages, SpringerVerlag, 1988.

[2] A. Bertoni, C. Choffrut, M. Goldwurm, and V. Lonati. On the number of occurrences of a symbol in words of regular languages. Theoret. Comput. Sci., 302(1-3):431-456, 2003.

[3] J. Bourdon and B. Vallée. Generalized pattern matching statistics. Mathematics and computer science II: algorithms, trees, combinatorics and probabilities. Proc. of Versailles Colloquium, Birkhauser, 249-265, 2002.

[4] F.G. Csàki. Some notes on the inversion of confluent Vandermonde matrices, IEEE Trans. Automat. Control, AC 20, 154-157, 1975. 
[5] D. de Falco, M. Goldwurm, V. Lonati. Frequency of symbol occurrences in bicomponent stochastic models. Theoret. Comput. Sci., 327 (3):269-300, 2004.

[6] P. Flajolet. Mathematical methods in the analysis of algorithms and data structures. In Trends in Theoretical Computer Science, E. Börger ed., Computer Science Press, 225-304, 1988.

[7] B.V. Gnedenko. The theory of probability (translated by G. Yankovsky). Mir Publishers - Moscow, 1976.

[8] M. Goldwurm, Probabilistic estimation of the number of prefixes of a trace, Theoret. Comp. Sci., 92:249-268, 1992.

[9] P. Grabner and M. Rigo. Additive functions with respect to numeration systems on regular languages. Monatshefte für Mathematik, 139: 205-219, 2003.

[10] L. J. Guibas and A. M. Odlyzko. Maximal prefix-synchronized codes. SIAM J. Appl. Math., 35:401-418, 1978.

[11] L. J. Guibas and A. M. Odlyzko. String overlaps, pattern matching, and nontransitive games. Journal of Combinatorial Theory. Series A, 30(2):183208, 1981.

[12] P. Henrici. Applied and computational complex analysis, vol. 1, John Wiley, 1974.

[13] P. Jokinen and E. Ukkonen. Two algorithms for approximate string matching in static texts. Proceedings MFCS'91, Lect. Notes in Comput. Sci., vol. n. 520, Springer, 1991, 240-248.

[14] P. Lancaster and M. Tismenetsky. The theory of matrices, Academic Press, 1985.

[15] U. Luther and K. Rost. Matrix exponentials and inversions of confluent Vandermonde matrices, Electronic Trans. on Numerical Analysis, vol. 18, 91100, 2004 .

[16] P. Nicodeme, B. Salvy, and P. Flajolet. Motif statistics. Theoret. Comput. Sci., 287(2):593-617, 2002.

[17] B. Prum, F. Rudolphe and E. Turckheim. Finding words with unexpected frequencies in deoxyribonucleic acid sequence. J. Roy. Statist. Soc. Ser. B, 57:205-220, 1995.

[18] M. Régnier and W. Szpankowski. On pattern frequency occurrences in a Markovian sequence. Algorithmica, 22 (4):621-649, 1998.

[19] A. Salomaa and M. Soittola. Automata-Theoretic Aspects of Formal Power Series, Springer-Verlag, 1978.

[20] R. Sedgewick and P. Flajolet. An introduction to the analysis of algorithms, Addison-Wesley, 1996. 
[21] E. Seneta. Non-negative matrices and Markov chains, Springer-Verlag, New York Heidelberg Berlin, 1981.

[22] E. Sutinen and W. Szpankowski. On the collapse of q-gram filtration, Proceedings of FUN with Algorithms, 178-193, Elba 1998. 